\title{
Intrinsic Deuterium Kinetic Isotope Effects in Glutamate Mutase Measured by an Intramolecular Competition Experiment**
}

\author{
Miri Yoon, Anastasia Kalli, Hyang-Yeol Lee, Kristina Håkansson, and E. Neil G. Marsh*
}

Kinetic isotope effects (KIEs) provide a powerful tool to interrogate transition states of both enzymic and non-enzymic reactions,${ }^{[1-4]}$ provided that one can measure the intrinsic KIE on the chemical step of interest, that is, the isotope effect undiminished by other isotope-insensitive steps that may contribute to the overall rate of reaction. For small molecules reacting in solution the KIE measured usually represents the intrinsic value; however, for enzymes this is seldom the case. Here we report the first measurement of the intrinsic KIE in an adenosylcobalamin enzyme (AdoCbl, coenzyme $\mathrm{B}_{12}$ ) for hydrogen atom transfer from substrate to coenzyme, which is a key step in the mechanism of this class of enzymes. For the $\mathrm{B}_{12}$ enzyme glutamate mutase the intrinsic deuterium KIE for hydrogen transfer from the substrate, $(2 S, 3 S)$-3-methylaspartate, to $5^{\prime}$-deoxyadensosine is 4.1 . This value is well within the semiclassical limit for a deuterium isotope effect and is much smaller than the anomalously large KIEs previously measured in other $\mathrm{B}_{12}$ enzymes and non-enzymatic model reactions, which were attributed to extensive hydrogen tunneling.

Glutamate mutase one is of a group of AdoCbl-dependent enzymes that catalyze unusual isomerization reactions that formally involve a 1,2 hydrogen atom migration and proceed through a mechanism involving carbon-based free radical intermediates (Scheme 1). ${ }^{[5-10]}$ Radicals are generated by homolysis of the reactive cobalt-carbon bond of the coenzyme to form cob(II)alamin, a cobalt(II) intermediate, and the 5'-deoxyadenosyl radical. The adenosyl radical then abstracts the migrating hydrogen from the substrate to form 5 '-deoxyadenosine and the substrate radical. The substrate radical next undergoes rearrangement to give the product radical, which is then quenched by hydrogen transfer from $5^{\prime}$ deoxyadenosine to give the product and regenerate the $5^{\prime}$ deoxyadenosyl radical. Finally, recombination of the adenosyl radical and cob(II)alamin to reform the coenzyme completes the catalytic cycle.

Our interest in the mechanisms by which enzymes generate free radicals, as exemplified by dependent glutamate mutase, ${ }^{[10]}$ led us to undertake an extensive set of KIE measurements to examine how hydrogen abstraction from the

[*] M. Yoon, A. Kalli, H.-Y. Lee, Prof. K. Håkansson, Prof. E. N. G. Marsh Department of Chemistry

University of Michigan

Ann Arbor, MI 48109-1055 (USA)

Fax: $(+1)$ 734-615-3790

E-mail:nmarsh@umich.edu

Homepage: http://www.umich.edu/ marshlab

[**; E.N.G.M. acknowledges the support of NIH grant GM R01 59227 which funded this work. Work in K.H.'s laboratory is in part supported by an Eli Lilly Analytical Chemistry Award.
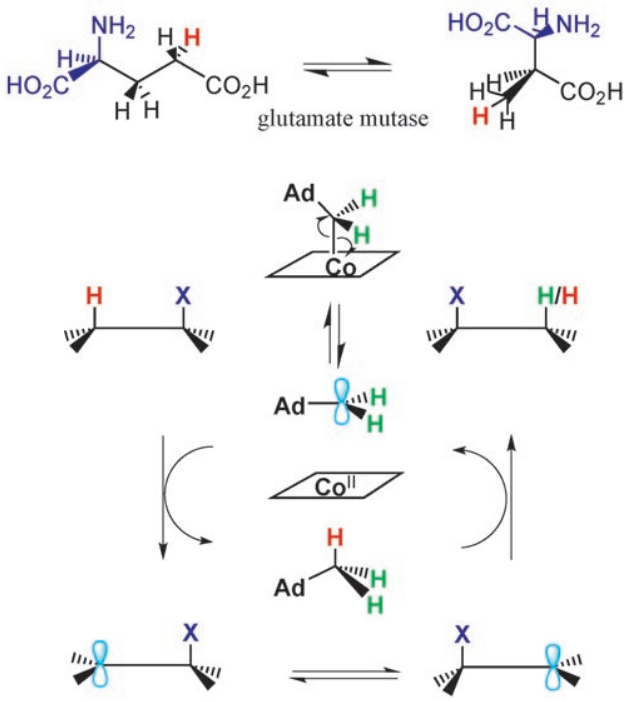

Scheme 1. Reaction catalyzed by glutamate mutase and a minimal mechanistic scheme for the rearrangements catalyzed by AdoCbldependent enzymes ( $\mathrm{X}=\mathrm{OH}, \mathrm{NH}_{2}$, or $\left.\mathrm{R}\right)$.

substrate and coenzyme homolysis are coupled together. ${ }^{[11-15]}$ KIE measurements using deuterium- and tritium-labeled substrates and coenzyme have proved especially informative probes of the key steps of $\mathrm{Co}^{-} \mathrm{C}$ bond homolysis and hydrogen atom abstraction from substrate. Pre-steady-state measurements on a number of enzymes have shown that hydrogen abstraction is kinetically coupled to $\mathrm{Co}-\mathrm{C}$ bond homolysis, ${ }^{[11,16-18]}$ as evidenced by the appearance of a kinetic isotope effect on cobalt-carbon bond homolysis when the enzymes are reacted with deuterated substrates.

This observation implies that the $5^{\prime}$-dA radical is a highenergy intermediate that only has a fleeting existence. Furthermore, the KIEs reported for several AdoCbl enzymes are extremely large (ranging from $\approx 10$ to $\approx 50$ ), which has generally been attributed to hydrogen tunneling. In particular, extensive hydrogen tunneling in methylmalonyl-CoA mutase has been deduced from the temperature dependence of the deuterium isotope effect on hydrogen transfer and $\mathrm{Co}^{-}$ C bond homolysis. ${ }^{[19]}$

The KIEs discussed above were all measured indirectly by UV/Vis stopped-flow spectroscopy, using the spectroscopic changes associated with $\mathrm{Co}^{-} \mathrm{C}$ bond homolysis as a convenient reporter of the kinetics. Although we originally reported a very large primary deuterium isotope effect, suggestive of tunneling, for the formation of cob(II)alamin and 5'-dA upon reaction of hologlutamate mutase with deuterated substrates $\left(k_{\mathrm{H}} / k_{\mathrm{D}}=28\right.$ with glutamate and 35 with methylaspartate $),{ }^{[1]}$ we have recently had cause to revise our interpretation of the 
stopped-flow data upon which these numbers were based. Experiments using rapid quenching techniques and mass spectral analysis to analyze the deuterium content of $5^{\prime}$-dA formed in the reaction as a function of time ${ }^{[14]}$ gave a value for the KIE of only 2.4.

The origin of this discrepancy lies in the fact that we misinterpreted the biphasic kinetics of AdoCbl homolysis observed by stopped-flow spectroscopy when glutamate mutase was reacted with deuterated substrates as rising from negative cooperativity between the two active sites of the dimeric enzyme. This explanation appeared reasonable because the two phases of the reaction had approximately equal amplitudes, and we had previously observed weakly cooperative binding of the monomeric $S$ subunit to the dimeric E subunit of the wild-type enzyme. ${ }^{[20]}$ Furthermore the KIEs we calculated were in general agreement with those reported for other AdoCbl-dependent enzymes. ${ }^{[18,19,21,22]}$ Only in our later experiments did it become apparent that the slower rate was associated with multiple turnovers of the deuterated substrates resulting in di- and trideuterated $5^{\prime}$-dA and that it arose from an unexpectedly large inverse equilibrium isotope effect shifting the equilibrium towards homolysis of AdoCbl.

Given the rather small deuterium KIE we measured for glutamate mutase in our later experiment, ${ }^{[14]}$ we suspected that, even though the measurement was made in the presteady state, the KIE might be suppressed by nonisotopically sensitive steps. We have therefore devised an experiment aimed at determining the intrinsic KIE for hydrogen atom transfer from the substrate, $(2 S, 3 S)-3$-methylaspartate, to $5^{\prime}$ dA catalyzed by glutamate mutase. The experiment takes advantage of the fact that for hydrogen transfer from methyl groups the intrinsic deuterium isotope effect can be measured by specifically labeling the methyl carbon with one or two deuterium atoms and analyzing the isotopic composition of the reaction products. ${ }^{[23]}$ The isotope effect can be measured, even when the isotopically sensitive step is not rate determining, because it is manifested through intramolecular competition between protium and deuterium atoms, which remain chemically equivalent even in the enzyme active site owing to the rapid rotation of the methyl group.

The principle of the experiment as implemented for glutamate mutase is shown in Scheme 2. The enzyme is

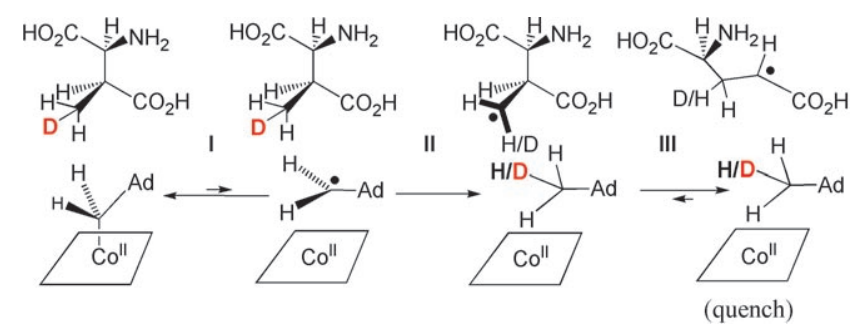

Scheme 2. Measurement of intrinsic KIE by intramolecular isotope competition using $\left[{ }^{2} \mathrm{H}_{1}\right]$ methylaspartate. Isotopic discrimination occurs in step II, and rapid rearrangement of methylaspartyl radical to glutamyl radical (step III) prevents scrambling of the deuterium between substrate and coenzyme on the short time scales employed in the experiment. reacted with methylaspartate specifically monodeuterated in the methyl group. During the reaction the 5 '-deoxyadenosyl radical, generated by homolysis of AdoCbl, is confronted with the choice of abstracting either protium or deuterium from the methyl group of the same substrate molecule. Hydrogen or deuterium abstraction generates methylaspartyl radical, which rapidly rearranges to the much more stable glutamyl radical so that at sufficiently short times $(<100 \mathrm{~ms})$ the reaction is effectively irreversible and the intrinsic isotope effect is manifested.

Glutamate mutase and $\left[{ }^{2} \mathrm{H}_{1}\right](2 S, 3 S)$-3-methylaspartate, regiospecifically monodeuterated in the methyl group, were prepared as described previously. ${ }^{[24,25]}$ Solutions containing $275 \mu \mathrm{M}$ glutamate mutase and $275 \mu \mathrm{M}$ AdoCbl in $50 \mathrm{~mm}$ phosphate buffer, pH 7.0, and $2.0 \mathrm{~mm}\left[{ }^{2} \mathrm{H}_{1}\right]$ methylaspartate in the same buffer (concentrations before mixing) were introduced into a rapid-quench flow apparatus maintained at $10^{\circ} \mathrm{C}$. The solutions were mixed in a $1: 1$ ratio and allowed to react for various times $(20-80 \mathrm{~ms})$ before quenching with $2.5 \%$ trifluoroacetic acid. The $5^{\prime}$-dA formed in the reaction was recovered by reverse-phase HPLC. ${ }^{[26]}$

The deuterium content of the $5^{\prime}$-dA was determined by ultrahigh-resolution Fourier transform ion cyclotron resonance mass spectrometry (FTICR MS). The 5'-dA isolated from the reaction contains a mixture of isotopomers, with contributions from natural abundance ${ }^{13} \mathrm{C},{ }^{15} \mathrm{~N},{ }^{17} \mathrm{O}$, and ${ }^{2} \mathrm{H}$ isotopes in addition to any deuterium from the substrate. Normally, the presence of ${ }^{13} \mathrm{C}$ complicates the analysis of deuterium KIEs because the peaks due to deuterated and ${ }^{13} \mathrm{C}$ containing molecules overlap. However, the resolution of an FTICR MS instrument is sufficiently high ${ }^{[27,28]}$ that peaks due to ${ }^{13} \mathrm{C}$-, ${ }^{2} \mathrm{H}$-, and ${ }^{15} \mathrm{~N}$-containing molecules are baseline resolved. This ultrahigh resolution has the advantage that the ${ }^{13} \mathrm{C}$ peak can now be used as an internal standard to measure the relative abundance of the peak due to ${ }^{2} \mathrm{H}$-labeled 5'-dA.

Figure 1 shows a representative mass spectrum from an experiment in which the reaction was stopped after $36 \mathrm{~ms}$ and the $5^{\prime}$-dA analyzed by electrospray FTICR MS in positive-ion mode. The $[M+\mathrm{H}]^{+}$peaks due to naturally occurring ${ }^{13} \mathrm{C}$ - and ${ }^{2} \mathrm{H}$-labeled $5^{\prime}$-dA molecules are baseline resolved and are of similar abundance. The intrinsic KIE can simply be calculated by integrating the peak areas for $\left[{ }^{12} \mathrm{C}\right] 5^{\prime}$-dA and $\left[{ }^{12} \mathrm{C},{ }^{2} \mathrm{H}\right] 5^{\prime}$-dA and comparing their ratio. Because there are two protium atoms and only one deuterium atom in the methyl group of methylaspartate, the KIE must be corrected by a factor of 2 . Alternatively, because the natural abundance of ${ }^{13} \mathrm{C}$ is known to great precision, the intrinsic $\mathrm{KIE}$ can also be calculated by comparing the ratio of $\left[{ }^{13} \mathrm{C}\right] 5^{\prime}-\mathrm{dA}$ to $\left[{ }^{12} \mathrm{C},{ }^{2} \mathrm{H}\right] 5^{\prime}-\mathrm{dA}$ and correcting the result for the natural abundance, $n$, using Equation (1).

$\mathrm{KIE}=\frac{1}{2} \frac{\left.\left[{ }^{13} \mathrm{C}\right] 5^{\prime}-\mathrm{dA}\right]}{\left[\left[{ }^{2} \mathrm{H}\right] 5^{\prime}-\mathrm{dA}\right]} \frac{(1-n)}{n}$

We calculated the KIE for hydrogen transfer from methylaspartate to $5^{\prime}$-dA for reaction times ranging from $28 \mathrm{~ms}$ to $78 \mathrm{~ms}$, as summarized in Table 1 . The KIEs calculated from either the ratio of $\left[{ }^{12} \mathrm{C}\right] 5^{\prime}-\mathrm{dA}$ to $\left[{ }^{2} \mathrm{H}\right] 5^{\prime}-\mathrm{dA}$ 


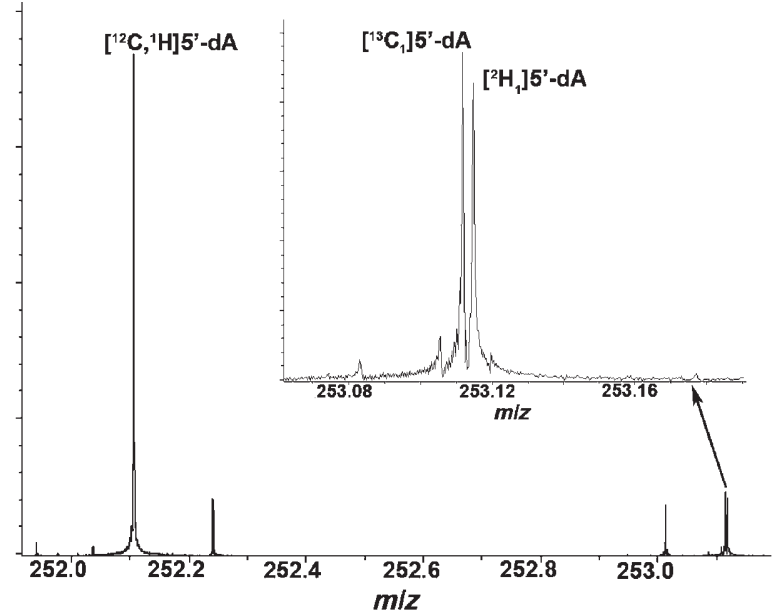

Figure 1. High-resolution electrospray (positive-ion mode) ICR MS of $5^{\prime}$-dA isolated from glutamate mutase after reaction with $\left[{ }^{2} \mathrm{H}_{1}\right]$ methylaspartate for $36 \mathrm{~ms}$. The peaks due to ${ }^{13} \mathrm{C},{ }^{2} \mathrm{H}$-labeled and unlabeled 5 '- $\mathrm{dA}$ are indicated. Inset: Expansion of the region around 253.115 amu showing the ${ }^{13} \mathrm{C}$-labeled and ${ }^{2} \mathrm{H}$-labeled ions clearly resolved (the small peak at 253.108 is due to natural-abundance $\left.\left[{ }^{15} \mathrm{~N}\right] 5^{\prime}-\mathrm{dA}\right)$.

Table 1: Intrinsic $\mathrm{KIEs}$ at $10^{\circ} \mathrm{C}$ for hydrogen transfer determined at various reaction times.

\begin{tabular}{llll}
\hline Reaction time $[\mathrm{ms}]$ & $\mathrm{KIE}\left({ }^{12} \mathrm{C} /{ }^{2} \mathrm{H}\right)^{[\mathrm{a}]}$ & $\operatorname{KIE}\left({ }^{13} \mathrm{C} /{ }^{2} \mathrm{H}\right)^{[\mathrm{b}]}$ & $n^{[\mathrm{c}]}$ \\
\hline 28 & $3.7 \pm 0.1$ & $3.9 \pm 0.3$ & 9 \\
37 & $4.4 \pm 0.3$ & $3.9 \pm 0.2$ & 9 \\
56 & $4.3 \pm 0.4$ & $4.0 \pm 0.4$ & 11 \\
76 & $4.1 \pm 0.2$ & $4.2 \pm 0.4$ & 3 \\
$\approx 1000$ & $0.95 \pm 0.04$ & $0.96 \pm 0.06$ & 4
\end{tabular}

[a] KIE determined from the ratio of $\left[{ }^{12} \mathrm{C}\right] 5^{\prime}-d A$ to $\left[{ }^{2} \mathrm{H}\right] 5^{\prime}-d A$. [b] KIE determined from the ratio of $\left[{ }^{13} \mathrm{C}\right] 5^{\prime}-d A$ to $\left[{ }^{2} \mathrm{H}\right] 5^{\prime}-d A$. [c] Number of determinations.

or $\left[{ }^{13} \mathrm{C}\right] 5^{\prime}-\mathrm{dA}$ to $\left[{ }^{2} \mathrm{H}\right] 5^{\prime}-\mathrm{dA}$ are, as expected, the same within experimental error. Furthermore, the value for the KIE does not change significantly over the timescale surveyed, suggesting that multiple transits of deuterium between $5^{\prime}$-dA and methylaspartate (which would increase deuterium incorporation into 5 '-dA and reduce the apparent KIE) do not occur on this timescale. The average value of the intrinsic deuterium $\mathrm{KIE}$ is $4.1 \pm 0.2$. A control experiment in which the substrate was allowed to react with the enzyme for approximately $1 \mathrm{~s}$ gave, as expected, a KIE of very close to 1 , indicating that at longer times equilibration of deuterium between coenzyme and substrate does indeed occur.

An important assumption in our experiment is that hydrogen and deuterium have the same probability of occupying the abstractable position in the methyl group of the substrate, in other words, that the methyl group can rotate completely freely. We note that a C-D bond is slightly shorter than a $\mathrm{C}-\mathrm{H}$ bond so even this most conservative of substitutions introduces a slight asymmetry into the methyl group which could, in principle, lead to the preference of one rotational conformation over another. However, we consider this possibility extremely unlikely as the differences in energies between such conformations are undoubtedly very small relative to thermal energies at room temperature. Indeed, we are aware of no enzyme where restricted rotation of a methyl group in the active site has been demonstrated.

Numerous deuterium and tritium isotope effect measurements have been reported for AdoCbl-dependent enzymes over many years. These measurements have been made in both the steady-state and pre-steady-state regime, and the values reported reflect to varying degrees the influence of nonisotopically sensitive steps. Most of the pre-steady-state measurements, though, report KIEs that are larger than expected for semiclassical behavior and that may reasonably be explained by tunneling. ${ }^{[18,19,21,22,29]}$ Recent KIE measurements that provide the most pertinent comparison to the present case are summarized in Table 2 . In striking contrast, the KIE of 4.1 we measure for glutamate mutase is much smaller and well within the range expected for semiclassical behavior.

Table 2: KIEs for hydrogen transfer measured for selected AdoCbldependent isomerases and model reactions.

\begin{tabular}{lll}
\hline Enzyme & $\mathrm{KIE}$ at $10^{\circ} \mathrm{C}$ & Ref. \\
\hline $\begin{array}{lll}\text { methylmalonyl-CoA mutase } \\
\text { ethanolamine ammonia lyase } \\
\text { glutamate mutase }\end{array}$ & 43 & {$[19]$} \\
$\begin{array}{l}\text { model reaction } \\
\text { thermolysis of AdoCbl in ethylene glycol }\end{array}$ & 4.1 & {$[18]$} \\
thermolysis of neopentylCbl in ethylene glycol & 39 & This study \\
\hline
\end{tabular}

Of particular interest is the work of Finke's group who measured the KIE for hydrogen abstraction in two nonenzymic model systems. ${ }^{[30,31]}$ Both experiments measured the kinetics of hydrogen abstraction from ethylene glycol (mimicking the diol dehydrase reaction) either by $5^{\prime}$-dA radical, generated by thermolysis of AdoCbl, or neopentyl radical, generated by thermolysis of neopentylcobalamin. In both systems significant hydrogen tunneling was detected from analysis of Arrhenius plots, and furthermore the KIEs measured were much larger than could be accounted for by semi-classical formulation of KIEs. The isotope effects on Arrhenius pre-exponential factors and activation energies measured in these model systems were very similar to those measured in methylmalonyl-CoA mutase,${ }^{[19]}$ as were the KIEs when corrected for differences in temperature. These results led to the conclusion that tunneling was an intrinsic property of both the enzyme catalyzed and non-enzymic reaction, and that there was no enhancement of tunneling by the enzyme, as has been proposed for other enzymes where tunneling has been investigated. ${ }^{[32-34]}$

Given that the KIEs for hydrogen abstraction in both the model systems and methylmalonyl-CoA mutase are unusually large, it is extremely surprising that the intrinsic KIE in glutamate mutase should be so much smaller. The hydrogen abstraction reactions catalyzed by methylmalonyl-CoA mutase and glutamate mutase are chemically identical, and, if anything, the stopped-flow technique used to measure the KIE in methylmalonyl-CoA mutase may underestimate the 
intrinsic KIE. Although $\left[{ }^{2} \mathrm{H}_{3}\right]$ methylmalonyl-CoA and $\left[{ }^{2} \mathrm{H}_{4}\right]$ ethylene glycol were used in the other experiments and therefore the primary KIE is likely inflated by a contribution from the secondary KIE, this effect could not explain such large differences between the isotope effects. We are not aware of any case where two such similar chemical reactions proceed with such different isotope effects.

The fact that the non-enzymic model reactions also proceed with such large KIEs argues that glutamate mutase must be modulating the transition state for hydrogen transfer in a manner that reduces the KIE associated with it. How this might be occurring is presently unclear. Although the intrinsic $\mathrm{KIE}$ is within semiclassical limits, this result does not rule out hydrogen tunneling in glutamate mutase. We have recently measured secondary tritium KIEs associated with the formation of $5^{\prime}$-dA that indicate hydrogen transfer occurs with both tunneling and significant coupling to the motion of the secondary hydrogen atoms. ${ }^{[13,15]}$ In such cases the primary KIE is reduced by the coupled motion of the secondary hydrogens, and the secondary KIE is correspondingly inflated. ${ }^{[35]}$ This phenomenon is best studied for NAD $(\mathrm{P}) \mathrm{H}$ dependent dehydrogenases that exhibit primary deuterium KIEs that are also relatively small, being at the upper limit of those expected semiclassically. ${ }^{[4]}$

Lastly we comment on the possibility, raised by one reviewer of this manuscript, that so-called "magnetic isotope effects" may play a role here. Magnetic isotope effects arise in radical reactions when the nuclear spin of the isotope couples to the spin of the unpaired electron. This can influence the rate of intersystem crossing between singlet and triplet states and thereby the rates of radical recombination reactions. Their influence on photochemical radical reactions is well documented. ${ }^{[36]}$ However, although magnetic field effects have been shown to influence the kinetics of coenzyme homolysis in the $\mathrm{B}_{12}$ enzyme ethanolamine ammonia lyase $^{[37,38]}$ (and other reactions involving the formation of radical pairs), there are no studies in which a magnetic isotope effect has been shown to operate in an enzyme-catalyzed reaction.

In conclusion, by taking advantage of intramolecular competition between hydrogen and deuterium atoms at the methyl group of methylaspartate we have measured, for the first time, the intrinsic primary deuterium isotope for $5^{\prime}$-dA formation in a $B_{12}$ enzyme. Remarkably, the KIE is much smaller than that expected based on KIE measurements on other $\mathrm{B}_{12}$ enzymes and model systems. This argues strongly for a role for glutamate mutase in modulating the transition state for hydrogen transfer and thereby changing the KIE. The result is in surprising contrast to those measured for methylmalonyl-CoA mutase and non-enzymic model systems which are both of similar magnitude and much larger than the semiclassical limit. Further KIE measurements are in progress to determine whether hydrogen tunneling makes an important contribution in glutamate mutase, as it appears to do in methylmalonyl-CoA mutase and in the model reactions.

\section{Experimental Section}

The synthesis of $\left[{ }^{2} \mathrm{H}_{1}\right]$ methyl- $(2 S, 3 S)$-methylaspartate and purification of glutamate mutase from recombinant $E$. coli have been described previously. ${ }^{[24,25]}$ All other reagents were of the highest quality commercially available.

Rapid quench experiments were performed at $10^{\circ} \mathrm{C}$ using a $\mathrm{Hi}$ Tech RQF-63 apparatus. A $73.5-\mu \mathrm{L}$ portion of $275 \mu \mathrm{M}$ glutamate mutase in $50 \mathrm{~mm}$ potassium phosphate buffer ( $\mathrm{pH}$ 7.0) was immediately mixed with $6.5 \mu \mathrm{L} 3.4 \mathrm{~mm}$ AdoCbl immediately before the experiment to reconstitute the holoenzyme. The holoenzyme mixture was allowed to react with $80 \mu \mathrm{L}$ of $2.2 \mathrm{~mm}\left[{ }^{2} \mathrm{H}_{1}\right]$ methylaspartate in $50 \mathrm{~mm}$ potassium phosphate buffer $(\mathrm{pH} 7.0)$. The reactions were quenched with $80 \mu \mathrm{L}$ of $2.5 \%$ trifluoroacetic acid, after aging for various reaction times (28-76 ms). 5'-Deoxyadenosine was recovered and quantified by $\mathrm{C}_{18}$ reverse-phase HPLC as described previously. ${ }^{[26]}$ All the procedures were conducted in dim red light. Samples of labeled $5^{\prime}-\mathrm{dA}$ were lyophilized and stored at $-20^{\circ} \mathrm{C}$ prior to mass spectrometric analysis.

Mass spectral analysis: 5'-dA was redissolved in solvent containing $50 \% \mathrm{MeOH}$ and $0.1 \%$ formic acid. Experiments were performed in positive-ion mode at $50 \mu \mathrm{Lh}^{-1}$ with a $7 \mathrm{~T}$ Q-FT-ICR mass spectrometer (Bruker Daltonics, Billerica, MA) as described previously. ${ }^{[39]}$ Data were collected in narrowband mode. The masses of $\left[{ }^{12} \mathrm{C}_{9},{ }^{1} \mathrm{H}_{13},{ }^{13} \mathrm{C}_{1},{ }^{14} \mathrm{~N}_{5},{ }^{16} \mathrm{O}_{3}\right] 5^{\prime}-\mathrm{dA}$ and $\left[{ }^{12} \mathrm{C}_{10},{ }_{1} \mathrm{H}_{12},{ }^{2} \mathrm{H}_{1},{ }^{14} \mathrm{~N}_{5},{ }^{16} \mathrm{O}_{3}\right] 5^{\prime}$-dA are $252.10436 \mathrm{Da}$ and $252.10799 \mathrm{Da}$, respectively, a mass difference of $3.6 \mathrm{mDa}$. The identities of the peaks due to unlabeled, ${ }^{13} \mathrm{C}$-labeled, and ${ }^{2} \mathrm{H}$-labeled $5^{\prime}$-dA were confirmed by examining a sample of commercially available $5^{\prime}$-dA under the same experimental conditions. Only the lower $\mathrm{m} / \mathrm{z}$ peak was observed for non-deuterated $5^{\prime}$-dA, whereas two peaks $\left({ }^{12} \mathrm{C}_{9},{ }^{1} \mathrm{H}_{13},{ }^{13} \mathrm{C}_{1},{ }^{14} \mathrm{~N}_{5},{ }^{16} \mathrm{O}_{3}\right.$ and ${ }^{12} \mathrm{C}_{10},{ }^{1} \mathrm{H}_{12},{ }^{2} \mathrm{H}_{1},{ }^{14} \mathrm{~N}_{5},{ }^{16} \mathrm{O}_{3}$ ) were seen following incubation in $1 \% \mathrm{D}_{2} \mathrm{O}$. The experimental masses of the enzymatic samples compare accurately $(<2 \mathrm{ppm})$ with the masses of the standards and the calculated theoretical masses. As an internal control, the instrument was tuned to ensure that the detected relative abundance of $\left[{ }^{12} \mathrm{C}\right] 5^{\prime}-\mathrm{dA}$ to $\left[{ }^{13} \mathrm{C}\right] 5^{\prime}-$ $\mathrm{dA}$ was as close as possible to the calculated ratio of 0.1325 .

Received: June 5, 2007

Published online: October 1, 2007

Keywords: cobalamins $\cdot$ coenzyme $B_{12} \cdot$ enzymes mass spectrometry $\cdot$ radicals

[1] W. W. Cleland, CRC Crit. Rev. Biochem. 1982, 13, 385-428.

[2] W. W. Cleland in Isotopes in Organic Chemistry, Vol. 7 (Eds.: E. Buncel, C. C. Lee), Elsevier, Amsterdam 1987.

[3] V. L. Schramm, Curr. Opin. Chem. Biol. 2001, 5, 556-563.

[4] Z. D. Nagel, J. P. Klinman, Chem. Rev. 2006, 106, 3095-3118.

[5] P. A. Frey, A. D. Hegerman, G. H. Reed, Chem. Rev. 2006, 106, 3302-3316.

[6] R. Banerjee, Chem. Rev. 2003, 103, 2083-2094.

[7] E. N. G. Marsh, C. L. Drennan, Curr. Opin. Chem. Biol. 2001, 5, 499-505.

[8] P. A. Frey, Annu. Rev. Biochem. 2001, 70, 121-148.

[9] R. Banerjee, Biochemistry 2001, 40, 6191-96198.

[10] E. N. G. Marsh, Bioorg. Chem. 2000, 28, 176-189.

[11] E. N. G. Marsh, D. P. Ballou, Biochemistry 1998, 37, 11864 11872.

[12] H.-W. Chih, E. N. G. Marsh, Biochemistry 2001, 40, 13060 13067.

[13] M.-C. Cheng, E. N. G. Marsh, Biochemistry 2004, 43, 2155-2158.

[14] M.-C. Cheng, E. N. G. Marsh, Biochemistry 2005, 44, 2686-2691.

[15] M. C. Cheng, E. N. G. Marsh, Biochemistry 2007, 46, 883-889.

[16] S. S. Licht, C. C. Lawrence, J. Stubbe, Biochemistry 1999, 38, $1234-1242$. 
[17] R. Padmakumar, R. Banerjee, Biochemistry 1997, 36, 3713 3718 .

[18] V. Bandarian, G. H. Reed, Biochemistry 2000, 39, 12069-12075.

[19] S. Chowdhury, R. Banerjee, J. Am. Chem. Soc. 2000, 122, $5417-$ 5418.

[20] D. E. Holloway, E. N. G. Marsh, J. Biol. Chem. 1994, 269, 20425-20430

[21] D. A. Weisblat, B. M. Babior, J. Biol. Chem. 1971, 246, 6064 6071.

[22] M. K. Essenberg, P. A. Frey, R. H. Abeles, J. Am. Chem. Soc. 1971, 93, 1242-1251.

[23] K. R. Iyer, J. P. Jones, J. F. Darbyshire, W. F. Trager, Biochemistry 1997, 36, 7136-7143.

[24] H.-Y. Lee, M. Yoon, E. N. G. Marsh, Tetrahedron 2007, 63, $4663-4668$.

[25] H. P. Chen, E. N. G. Marsh, Biochemistry 1997, 36, 1493914945.

[26] H. W. Chih, E. N. G. Marsh, Biochemistry 1999, 38, $13684-$ 13691.

[27] F. He, C. L. Hendrickson, A. G. Marshall, Anal. Chem. 2001, 73, 647-650.
[28] S. D. H. Shi, C. L. Hendrickson, A. G. Marshall, Proc. Natl. Acad. Sci. USA 1998, 95, 11532-11537.

[29] E. N. G. Marsh, Biochemistry 1995, 34, 7542-7547.

[30] K. M. Doll, B. R. Bender, R. G. Finke, J. Am. Chem. Soc. 2003, 125, 10877-10884.

[31] K. M. Doll, R. G. Finke, Inorg. Chem. 2003, 42, 4849-4856.

[32] L. Masgrau, A. Roujeinikova, L. O. Johannissen, P. Hothi, J. Basran, K. E. Ranaghan, A. J. Mulholland, M. J. Sutcliffe, N. S. Scrutton, D. Leys, Science 2006, 312, 237-241.

[33] M. J. Sutcliffe, N. S. Scrutton, Eur. J. Biochem. 2002, 269, 30963102.

[34] A. Kohen, R. Cannio, S. Bartolucci, J. P. Klinman, Nature 1999, 399, 496-499.

[35] J. Suhnel, R. L. Schowen in Enzyme Mechanism from Isotope Effects (Ed.: P. F. Cook), CRC, Boca Raton, FL, 1991, pp. 3-35.

[36] N. J. Turro, Acc. Chem. Res. 2000, 33, 637-646.

[37] T. T. Harkins, C. B. Grissom, Science 1994, 263, 958-960.

[38] C. B. Grissom, Chem. Rev. 1995, 95, 3-24.

[39] J. Yang, J. J. Mo, J. T. Adamson, K. Hakansson, Anal. Chem. 2005, 77, 1876-1882. 